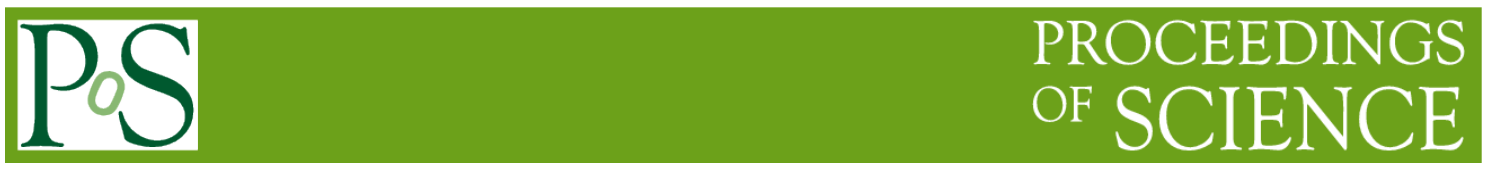

\title{
An Approach for Multi-attribute Group Decision Making under the Linguistic Environment
}

\author{
Ji-Bin Lan ${ }^{1}$, Meng-Yao Ning ${ }^{2 a}$, Yu-Wen Chen $^{3}$, Zhong-Xing Wang ${ }^{4}$ \\ College of Mathematics and Information Science \\ Guangxi University, Nanning, Guangxi 530004, China \\ E-mail: ${ }^{a} n m y-90163$. com
}

In this paper, a conversion relationship between values in interval $[0, g]$ and elements in the linguistic multiset is proposed and a linguistic aggregation model is introduced. Based on the conversion relationship and the model, a linguistic weighted aggregation $L W A_{\varphi}$ operator under the linguistic environment is established and its properties are studied. While the proposed aggregation operator is employed to deal with the multi-attribute group decision making problems. Finally, an illustrative example is provided and comparisons are made between the proposed method and the other existing methods.

ISCC2015

18-19, December 2015

Guangzhou, China

\footnotetext{
${ }^{1}$ Speaker

${ }^{2}$ Correspongding Author

${ }^{3}$ This study is supported by the National Foundation of China (No.7126100)

${ }^{4}$ This study is supported by the Youth Science Foundation Project of China

(No.71201037)
} 


\section{Introduction}

Multiple attribute group decision-making (MAGDM) is a typical decision problem, which has been widely applied to many areas such as industry, commerce and society, etc. In the real lift, because of complexity and uncertainty of the objective thing and the fuzziness of human beings, people tend to use linguistic terms to express their preferences [1]. For example, when evaluating the "price" of a TV, such linguistic terms as "high", "fair" and "low" are usually used.

In the linguistic MAGDM process, aggregating each decision maker's opinion into the group's opinion is the key [2]. Up still now, different linguistic models have been proposed to aggregate the linguistic information.

(1)The approximate model based on the extension principle [3]. Based on the membership functions, this model transforms the linguistic assessment information into the fuzzy numbers [4]; however, it is too hard to determine the accurate membership functions and it will cause loss of information during the transformation process[5].

(2)The symbolic model based on the ordinal scales [6].This model makes computations on the subscripts of linguistic terms and easy to operator [7]; however, the aggregation results is obtained according to an approximation process that makes it in shortage of accuracy [8].

(3)The 2-tuple linguistic fuzzy representation model [9]. This model first converts the linguistic assessment information into the 2-tuple linguistic variables, and then aggregate information based on some operators. The main advantage of this model is that it can avoid the information loss in linguistic information processing; however, Martinez and Herrera have said that the 2-tuple linguistic fuzzy representation model is only suitable for linguistic variables with equidistant labels [9]. In order to overcome this limitation, many researchers have made deep research. Herrera proposed a fuzzy linguistic methodology to deal with unbalanced linguistic term sets [10]. Wang and Hao proposed the 2-tuple proportional model [11]. Abchir developed the 2-tuple semantic model and the generalized symbolic modifiers [12]; however, $\mathrm{Xu}$ pointed out that the model was a bit of inconvenient in calculation [13].

(4) The model that computes with words directly, uses some operators to compute with words directly [14]. For example, $\mathrm{Xu}$ proposed the linguistic weighted arithmetic average (LWAA) operator [15], linguistic generalized (LGA) operator, linguistic hybrid aggregation (LHGA) operator [16] and Linguistic weights averaging $\left(\mathrm{LWA}_{2}\right)$ operator [17], etc. Zhou proposed linguistic power aggregation (LPA) operator [18]. Merigó developed induced linguistic generalized ordered weighted averaging (ILGOWA) operator [19]; moreover, many Linguistic MAGDM problems have been solved based on these operators. This model is straightforward and very convenient to calculate; however, it may have the aggregation results of linguistic information out of the original linguistic term set.

To overcome the above limitations, we proposed an approach for the linguistic MADGM problems. The paper is organized as follows. In Section 2, some basic concepts are briefly described and a new conversion relationship is provided. In Section 3, a linguistic weighted aggregation $L W A_{\varphi}$ operator is proposed and its properties are studied. In Section 4, an approach for the linguistic MAGDM problems is proposed. In Section 5, an illustrative example is developed and comparisons are made between the proposed method and the other existing 
methods, which can show the feasibility and applicability of the proposed approach. In Section 6 , the conclusion is made.

\section{Conversion Relationship}

In this section, some basic concepts are briefly described and a new conversion relationship is provided.

Suppose that $L=\left\{l_{i}=0,1, \cdots, g\right\}(g=2 \mathrm{k}, k \in N)$ be a finite and totally ordered discrete term set, where $l_{i}$ represents a possible value for a linguistic variable and $N$ is a set of non-negative integers [20-21]. Usually, any element of the set $L$ must satisfy four characteristics as follows [21]:

(1) The set is ordered: $l_{i} \leq l_{j}$ if $i \leq j$;

(2) There is a negation operator: neg $\left(l_{i}\right)=l_{j}$ such that $i=g-j$;

(3) Maximization operator: $\max \left\{l_{i}, l_{j}\right\}=l_{i}$ if $i \geq j$;

(4) Minimization operator: $\min \left\{l_{i}, l_{j}\right\}=l_{i}$ if $i \leq j$.

In order to keep all the given information, $L$ is extended to a continuous linguistic term set $\tilde{L}=\left\{l_{x} \mid x \in[0, g]\right\} \quad$ [15]. If $l_{x} \in L, l_{x}$ is called an original linguistic term; if $l_{x}\left(l_{x} \notin L\right), l_{x}$ is called a virtual linguistic term. Usually, the linguistic term $l_{x}\left(l_{x} \in L\right)$ is given by the decision maker, while the virtual linguistic term $l_{x}\left(l_{x} \notin L\right)$ only appears in computation [14].

Definition 1. For any $l_{x_{1}}, l_{x_{2}} \in \tilde{L}$, a binary relation $\preceq$ on $\tilde{L}$ is defined by

$$
l_{x_{1}} \preceq l_{x_{2}} \Leftrightarrow x_{1} \leq x_{2} \text { and } l_{x_{1}}=l_{x_{2}} \Leftrightarrow x_{1} \leq x_{2} \text {. }
$$

Property 1 . For any $l_{x_{1}}, l_{x_{2}}, l_{x_{3}} \in \tilde{L}$, the binary relation $\preceq$ satisfies the following three properties: (1) (reflexive): $l_{x_{1}} \preceq l_{x_{1}}$;

(2) (antisymmetry): $l_{x_{1}} \preceq l_{x_{2}}$ and $l_{x_{2}} \preceq l_{x_{1}}$ implies $l_{x_{1}}=l_{x_{2}}$;

(3) (transitivity): $l_{x_{1}} \preceq l_{x_{2}}$ and $l_{x_{2}} \preceq l_{x_{3}}$ implies $l_{x_{1}} \preceq l_{x_{3}}$.

Definition 2.[6] Let $\tilde{L}$ be the extended continuous linguistic term set, $\alpha_{x}=R^{+}\left(R^{+}\right.$ $=r \mid r \geq 0, r \in R)$ be a numeric value, $\tilde{u}: \tilde{L} \rightarrow R^{+}$is a mapping. For any $l_{x} \in \tilde{L}, \tilde{u}$ is defined by

$$
\tilde{u}\left(l_{x}\right)=\alpha_{x}
$$

where $0 \leq \alpha_{0}<\alpha_{1}<\cdots<\alpha_{g} . \tilde{u}$ is called the linguistic scale function.

Clearly, $\tilde{u}$ is a strictly monotonically increasing continuous function. The inverse function of $\tilde{u}$ exists, which is denoted by $\tilde{u}^{-1}$.

To date, several linguistic scale functions have been proposed. Such as Saaty scale, Exponential scale, power sacle [17].

Definition 3. Let $L=\left\{l_{i}=0,1, \cdots, g\right\}$ be a linguistic term set and $I=[0, g]$, the linguistic multiset $L_{r}$ is defined by

$$
L_{r}=L \times I=\left\{\left(l_{\text {round }(x)}, x\right) \mid x \in[0, g]\right\}
$$

where round (.) is the usual round operation. 
Definition 4. Suppose that linguistic multiset $L_{r}=\left\{\left(l_{\text {round }(x)}, x\right) \mid x \in[0, g]\right\}$ and $\varphi: \tilde{L} \rightarrow L_{r}$ be a mapping. For any $l_{x} \in \tilde{L}, \varphi$ is defined by

$$
\varphi\left(l_{x}\right)=\left(l_{\text {round }(x)}, x\right)
$$

where round (.) is the usual round operation.

Definition 5. For any $\left(l_{\text {round }\left(x_{1}\right)}, x_{1}\right),\left(l_{\text {round }\left(x_{2}\right)}, x_{2}\right) \in L_{r}$, a binary relation $\leq_{\varphi}$ on $L_{r}$ is defined by

$$
\left(l_{\text {round }\left(x_{1}\right)}, x_{1}\right) \leq_{\varphi}\left(l_{\text {round }\left(x_{2}\right)}, x_{2}\right) \Leftrightarrow x_{1} \leq x_{2}, \quad\left(l_{\text {round }\left(x_{1}\right)}, x_{1}\right)=_{\varphi}\left(l_{\text {round }\left(x_{2}\right)}, x_{2}\right) \Leftrightarrow x_{1}=x_{2}
$$

where $x_{1}, x_{2} \in[0, g]$.

For any $l_{x_{1}}, l_{x_{2}} \in \tilde{L}, \quad l_{x_{1}} \neq l_{x_{2}}$, we have $x_{1} \neq x_{2}$. So $\varphi\left(l_{x_{1}}\right) \neq \varphi\left(l_{x_{2}}\right)$. For any, $\left(l_{\text {round }(x)}, x\right) \in L_{r}$, there is only $l_{x} \in \tilde{L}$ so that $\varphi\left(l_{x}\right)=\left(l_{\text {round }(x)}, x\right)$. Thus the inverse mapping of $\varphi$ exists, which is denoted by $\varphi^{-1}$.

Suppose that $f$ be a monotone increasing and continuous function $f(x)=\alpha_{x}$ $\left(\alpha_{x} \in R^{+}\right)$. The inverse function of $f$ exists, which is denoted by $f^{-1}$. From the discussion above, we obtain the conversion relationship as follows:

$$
x \in[0, g] \underset{f^{-1}}{\stackrel{f}{\rightleftarrows}} \alpha_{x} \in R^{+} \underset{\tilde{u}}{\stackrel{\tilde{u}^{-1}}{\rightleftarrows}} l_{x} \in \tilde{L} \underset{\varphi^{-1}}{\stackrel{\varphi}{\rightleftarrows}}\left(l_{\text {round }(x)}, x\right) \in L_{r}
$$

Figure 1 : the conversion relationship between $x \in\lfloor 0, g\rfloor$ and $\left(l_{\text {round }(x)}, x\right) \in L_{r}$.

In fact, as to any $x_{1}, x_{2} \in[0, g]$,

$$
x_{1} \leq x_{2} \Leftrightarrow \alpha_{x_{1}} \leq \alpha_{x_{2}} \Leftrightarrow l_{x_{1}} \preceq l_{x_{2}} \Leftrightarrow \varphi\left(l_{x_{1}}\right) \preceq \varphi\left(l_{x_{2}}\right)
$$

$l_{\text {round }(x)}$ has the closest index label to $l_{x}$ and $x$ is the value that supports the ordering relation.

\section{A New Linguistic Aggregation Operator}

In this section, a linguistic weighted aggregation $L W A_{\varphi}$ operator is developed and its properties are studied.

Definition 6. Let $\tilde{L}=\left\{l_{x} \mid x \in[0, g]\right\}$ be a extended linguistic term set and $d: \tilde{L} \times \tilde{L} \rightarrow R^{+}$be a binary function. For any $l_{x_{1}}, l_{x_{2}} \in \tilde{L}, d$ which is defined by

$$
d\left(l_{x_{1}}, l_{x_{2}}\right)=\left|\tilde{u}\left(l_{x_{1}}\right)-\tilde{u}\left(l_{x_{2}}\right)\right|
$$

where $\tilde{u}\left(l_{x}\right)=\alpha_{x}\left(\alpha_{x} \in R^{+}\right)$.

In the group decision making, $\left(l_{x_{1}}, l_{x_{2}}, \cdots, l_{x_{n}}\right) \in L^{n}$ is the decision information for the alternative $A$ given by the decision makers $\left\{e_{1}, e_{2}, \cdots, e_{n}\right\}$, and $w=\left(w_{1}, w_{2}, \cdots, w_{n}\right)^{T}$ is the weighting vector of $\left(l_{x_{1}}, l_{x_{2}}, \cdots, l_{x_{n}}\right)$ satisfying $w_{i}>0, \sum_{i}^{n} w_{i}=1$. In order to aggregate the group's opinion, we consider the following mathematical programming model:

$$
(M 1) \min _{l_{x} \in \tilde{L}} F\left(l_{x}\right)=\sum_{i=1}^{n} w_{i}\left(d\left(l_{x_{i}}, l_{x}\right)\right)^{2}=\sum_{i=1}^{n} w_{i}\left(\tilde{u}\left(l_{x_{i}}\right)-\tilde{u}\left(l_{x}\right)\right)^{2}
$$

where $x \in[0, g]$. 
Let $h\left(\tilde{u}\left(l_{x}\right)\right)=\sum_{i=1}^{n} w_{i}\left(\tilde{u}\left(l_{x_{i}}\right)-\tilde{u}\left(l_{x}\right)\right)^{2}$

$$
=\tilde{u}^{2}\left(l_{x}\right)-2 \sum_{i=1}^{n} w_{i}\left(\tilde{u}\left(l_{x_{i}}\right)\right) \tilde{u}\left(l_{x}\right)+\sum_{i=1}^{n} w_{i} \tilde{u}^{2}\left(l_{x_{i}}\right)
$$

Obviously, $h$ is a quadratic function of $\tilde{u}\left(l_{x}\right)$, the axis of symmetry about $h\left(\tilde{u}\left(l_{x}\right)\right)$ is $\tilde{u}\left(l_{\bar{x}}\right)=\sum_{i=1}^{n} w_{i} \tilde{u}\left(l_{x_{i}}\right)$ and $\min _{l_{x} \in \tilde{L}} h\left(\tilde{u}\left(l_{x}\right)\right)=h\left(\tilde{u}\left(l_{\bar{x}}\right)\right)$; moreover, $l_{\bar{x}}=\tilde{u}^{-1}\left(\sum_{i=1}^{n} w_{i} \tilde{u}\left(l_{x_{i}}\right)\right)$.

According to the conversion relationship above, we have $\bar{x}=f^{-1}\left(\sum_{i=1}^{n} w_{i} \tilde{u}\left(l_{x_{i}}\right)\right)$.

Inference 1. The optimal solution of $(M 1)$ is $l_{\bar{x}}=l_{f^{-1}\left(\sum_{i=1}^{n} w_{i} \tilde{u}\left(l_{x}\right)\right)}$.

Moreover, $l_{f^{-1}\left(\sum_{i=1}^{n} w_{i} \tilde{u}\left(l_{x_{i}}\right)\right)}$ can be converted to

$$
\varphi\left(l_{f^{-1}\left(\sum_{i=1}^{n} w_{i} \tilde{u}\left(l_{x}\right)\right)}\right)=\left(l_{\text {round }\left(f^{-1}\left(\sum_{i=1}^{n} w_{i} \tilde{u}\left(l_{x}\right)\right)\right)}, f^{-1}\left(\sum_{i=1}^{n} w_{i} \tilde{u}\left(l_{x_{i}}\right)\right)\right) .
$$

Form the above discussion, linguistic information can be aggregated as follows:

Definition 7. An linguistic weighted aggregation $L W A_{\varphi}$ operator of dimension $n$ is a mapping $L W A_{\varphi}: L^{n} \rightarrow L_{r}$, furthermore,

$$
L W A_{\varphi}\left(l_{x_{1}}, l_{x_{2}}, \cdots, l_{x_{n}}\right)=\left(l_{\text {round }\left(f^{-1}\left(\sum_{i=1}^{n} w_{i} \tilde{u}\left(l_{x_{i}}\right)\right)\right.}, f^{-1}\left(\sum_{i=1}^{n} w_{i} \tilde{u}\left(l_{x_{i}}\right)\right)\right)
$$

where $w=\left(w_{1}, w_{2}, \cdots, w_{n}\right)^{T}$ be the weight vector of $\left(l_{x_{1}}, l_{x_{2}}, \cdots, l_{x_{n}}\right)$ satisfying $w_{i}>0, \sum_{i=1}^{n} w_{i}=1, \tilde{u}\left(l_{x}\right)=\alpha_{x}\left(\alpha_{x} \in R^{+}\right), f^{-1}\left(\alpha_{x}\right)=x(x \in[0, g])$.

In fact $L W A_{\varphi}\left(l_{x_{1}}, l_{x_{2}}, \cdots, l_{x_{n}}\right)=\varphi\left(l_{f^{-1}\left(\sum_{i=1}^{n} w_{i} \tilde{u}\left(l_{x}\right)\right)}\right)$.

Based on the binary relation $\leq_{\varphi}$ on $L_{r}$, we define the following ordering relation $\leq_{\psi}$ on $L^{n}$ to rank the alternatives:

Definition 8. Let $L=\left(l_{0}, l_{1}, \cdots, l_{g}\right)$ be a linguistic term set. For any $\left(l_{x_{1}}, l_{x_{2}}, \cdots, l_{x_{n}}\right),\left(l_{y_{1}}, l_{y_{2}}, \cdots, l_{y_{n}}\right) \in L^{n}$ a binary relation $\leq_{\psi}$ is defined by

$$
\begin{aligned}
& \left(l_{x_{1}}, l_{x_{2}}, \cdots, l_{x_{n}}\right) \leq_{\psi}\left(l_{y_{1}}, l_{y_{2}}, \cdots, l_{y_{n}}\right) \Leftrightarrow \varphi\left(l_{f^{-1}\left(\sum_{i=1}^{n} w_{i} \tilde{u}\left(l_{x}\right)\right)}\right) \leq_{\varphi} \varphi\left(l_{f^{-1}\left(\sum_{i=1}^{n} w_{i} \tilde{u}\left(l_{y_{1}}\right)\right)}\right) \\
& \left(l_{x_{1}}, l_{x_{2}}, \cdots, l_{x_{n}}\right)=_{\psi}\left(l_{y_{1}}, l_{y_{2}}, \cdots, l_{y_{n}}\right) \Leftrightarrow \varphi\left(l_{f^{-1}\left(\sum_{i=1}^{n} w_{i} \tilde{u}\left(l_{x_{1}}\right)\right)}\right)={ }_{\varphi} \varphi\left(l_{f^{-1}\left(\sum_{i=1}^{n} w_{i} \tilde{u}\left(l_{y_{1}}\right)\right)}\right)
\end{aligned}
$$

$l_{\text {round }\left(f^{-1}\left(\sum_{i=1}^{n} w_{i} \tilde{u}\left(l_{x}\right)\right)\right.} \in L$ is the aggregation result of $\left(l_{x_{1}}, l_{x_{2}}, \cdots, l_{x_{n}}\right)$ and $f^{-1}\left(\sum_{i=1}^{n} w_{i} \tilde{u}\left(l_{x_{i}}\right)\right)$ is the value supporting of the ordering relation.

Property 2. Let $\left(l_{x_{1}}, l_{x_{2}}, \cdots, l_{x_{n}}\right),\left(l_{y_{1}}, l_{y_{2}}, \cdots, l_{y_{n}}\right)$ be two linguistic argument collections, then the operator satisfies:

(1)(Monotonic)If $l_{x_{i}} \preceq l_{x_{j}}$ for all $i$, then

$$
L W A_{\varphi}\left(l_{x_{1}}, l_{x_{2}}, \cdots, l_{x_{n}}\right) \preceq L W A_{\varphi}\left(l_{y_{1}}, l_{y_{2}}, \cdots, l_{y_{n}}\right) .
$$

(2)(Bounded) For any weighting vector $w=\left(w_{1}, w_{2}, \cdots, w_{n}\right)^{T}$, the following inequality holds: $\min \left(l_{x_{i}} \preceq_{c_{i}}\right) \quad L W A_{\varphi}\left(l_{x_{1}}, l_{x_{2}}, \cdots \preceq_{x_{n}}\right) \quad \max \left(l_{x_{i}}, x_{i}\right)$.

(3)(Idempotent) If $l_{x_{i}}=l$ for all $i$, then $L W A_{\varphi}\left(l_{x_{1}}, l_{x_{2}}, \cdots, l_{x_{n}}\right)=l$. 
(4)(Commutative) If $w=(1 / n, \cdots, 1 / n)^{T}, L W A_{\varphi}\left(l_{x_{1}}, \cdots, l_{x_{n}}\right)=L W A_{\varphi}\left(l_{y_{1}}, \cdots, l_{y_{n}}\right)$.

(5)(Focus effect) If $w_{t}$ is sufficiently close to 1 and $l_{x_{t}} \prec l_{y_{t}}$, then

$$
L W A_{\varphi}\left(l_{x_{1}}, l_{x_{2}}, \cdots, l_{x_{n}}\right) \prec L W A_{\varphi}\left(l_{y_{1}}, l_{y_{2}}, \cdots, l_{y_{n}}\right) \text {. }
$$

Proof. the proofs are obvious and thus omitted.

Inference2. Let $\left(l_{x_{1}}, l_{x_{2}}, \cdots, l_{x_{n}}\right),\left(l_{y_{1}}, l_{y_{2}}, \cdots, l_{y_{n}}\right) \in L^{n}$,

If $f^{-1}\left(\sum_{i=1}^{n} w_{i} \tilde{u}\left(l_{x_{i}}\right)\right) \leq f^{-1}\left(\sum_{i=1}^{n} w_{i} \tilde{u}\left(l_{y_{i}}\right)\right)$. Then

$$
L W A_{\varphi}\left(l_{x_{1}}, l_{x_{2}}, \cdots, l_{x_{n}}\right) \preceq L W A_{\varphi}\left(l_{y_{1}}, l_{y_{2}}, \cdots, l_{y_{n}}\right) .
$$

Proof. By Eq. (2.6) the proof is obvious and thus omitted.

\section{An Approach for Multi-attribute Group Decision Making under Linguistic Environment}

Considering a MAGDA problem with linguistic information, let $A=\left\{A_{1}, A_{2}, \cdots, A_{m}\right\}$ be the discrete set of alternatives, $C=\left\{C_{1} C_{2}, \cdots, C_{n}\right\}$ be the set of attributes and $E=\left(e_{1}, e_{2}, \cdots, e_{t}\right)$ be the set of decision makers. Let $w=\left(w_{1}, w_{2}, \cdots, w_{n}\right)^{T}$ be the weight vector of attributes satisfying $w_{j} \in[0,1], \sum_{j=1}^{n} w_{j}=1$. And let $v=\left(v_{1}, v_{2}, \cdots, v_{t}\right)^{T}$ be the weight vector of decision makers satisfying $v_{k} \in[0,1], \sum_{k=1}^{t} v_{k}=1$. Assume each decision makers $e_{k} \in E(k=1,2, \cdots, t)$ provides his linguistic decision matrix $M_{k}=\left(l_{i j}^{k}\right)_{m \times n}$, where $l_{i j}^{k} \in L(i=1,2, \cdots, m ; j=1,2, \cdots, n)$ are given by $e_{k} \in E$ for the alternative $A_{i} \in A$ with respect to the attribute $C_{j} \in C$.

Then, using the $L W A_{\varphi}$ operator to aggregate all decision maker's linguistic decision information into a group's decision matrix $G$ :

$$
G=\left[\begin{array}{cccc}
\left(l_{r 11}, g_{11}\right) & \left(l_{r 12}, g_{12}\right) & \cdots & \left(l_{r 1 n}, g_{1 n}\right) \\
\left(l_{r 21}, g_{21}\right) & \left(l_{r 22}, g_{22}\right) & \cdots & \left(l_{r 2 n}, g_{2 \mathrm{n}}\right) \\
\vdots & \vdots & \cdots & \vdots \\
\left(l_{r m 1}, g_{m 1}\right) & \left(l_{r m 2}, g_{m 2}\right) & \cdots & \left(l_{r m n}, g_{m n}\right)
\end{array}\right]
$$

where

$$
\left(l_{r i j}, g_{i j}\right)=L W A_{\varphi}\left(l_{i j}^{1}, l_{i j}^{2}, \cdots, l_{i j}^{t}\right)=\left(l_{\text {round }\left(f^{-1}\left(\sum_{i=1}^{m} v_{k} \tilde{u}\left(l_{i j}^{k}\right)\right)\right)}, f^{-1}\left(\sum_{i=1}^{m} v_{k} \tilde{u}\left(l_{i j}^{k}\right)\right)\right)
$$

and $v_{k}$ is the weight of the decision maker $e_{k}$.

Therefore, $G$ corresponds to the group's comprehensive linguistic decision matrix $\tilde{G}$ and the value supporting matrix $\tilde{G}_{S}$ as follows:

where

$$
\tilde{G}=\left[\begin{array}{cccc}
l_{r 11} & l_{r 12} & \cdots & l_{r 1 n} \\
l_{r 21} & l_{r 22} & \cdots & l_{r 2 n} \\
\vdots & \vdots & \cdots & \vdots \\
l_{r m 1} & l_{r m 2} & \cdots & l_{r m n}
\end{array}\right] \quad, \quad \tilde{G}_{S}=\left[\begin{array}{cccc}
g_{11} & g_{12} & \cdots & g_{1 \mathrm{n}} \\
g_{21} & g_{22} & \cdots & g_{2 \mathrm{n}} \\
\vdots & \vdots & \cdots & \vdots \\
g_{m 1} & g_{m 2} & \cdots & g_{m n}
\end{array}\right]
$$




$$
r i j=\operatorname{round}\left(f^{-1}\left(\sum_{i=1}^{m} v_{k} \tilde{u}\left(l_{i j}^{k}\right)\right)\right), g_{i j}=f^{-1}\left(\sum_{i=1}^{m} v_{k} \tilde{u}\left(l_{i j}^{k}\right)\right), j=1,2, \cdots, n, k=1,2, \cdots, t \text {. }
$$

Furthermore, based on the value supporting matrix $\tilde{G}_{S}$ we can calculate the value supporting $N_{A_{i}}(i=1,2, \ldots, m)$ of each alternative by the weighted average method:

$$
N_{A_{i}}=\sum_{j=1}^{n} w_{j} g_{i j}, i=1,2, \ldots, m \text {. }
$$

Moreover, we can calculate the overall assessment $L_{A_{i}}(i=1,2, \ldots, m)$ of each alternative by Fig.1.

$$
L_{A_{i}}=\left(l_{\text {round }\left(N_{A}\right)}, N_{A_{i}}\right)
$$

Then the alternatives $A_{i}(i=1,2, \ldots, m)$ can be ranked based on the $N_{A_{i}}$.

To get the best alternative(s), the follows steps are involved:

Step1. Choose the function $\tilde{u}$ and $f^{-1}$.

Step2. Calculate the group's decision matrix $G$ by Eq.(4.1). Obtain the group's comprehensive linguistic decision matrix $\tilde{G}$ and the value supporting matrix $\tilde{G}_{S}$.

Step3. Calculate the value supporting $N_{A_{i}}$ and the overall assessment $L_{A_{i}}$ of each alternative by Eq. (4.4-4.5).

Step4. Rank all the alternatives based on the value supporting $N_{A_{i}}$ by Eq. (2.6) and obtain the best alternative(s).

\section{Illustrative Example}

Let us suppose an investment company, which wants to invest a sum of money in the best option (adapted from [21]). There is a panel with five possible alternatives to invest the money: $A_{1}$ is a car industry, $A_{2}$ is a food company, $A_{3}$ is a computer company, $A_{4}$ is an arms company, $A_{5}$ is a TV company. The investment company considers the following four attributes (with the weighting vector as $w=(0.35,0.15,0.20,0.30)^{T}$ ): $C_{1}$ is the risk analysis, $C_{2}$ is the growth analysis, $C_{3}$ is the social-political impact analysis, $C_{4}$ is the environmental impact analysis. The five possible alternatives $A_{i}(i=1,2, \ldots, 5)$ are to be evaluated by using the linguistic term set

$$
\begin{aligned}
L=\{ & \left\{l_{0}=\text { extremely poor }, l_{1}=\text { very poor }, l_{2}=\text { poor }, l_{3}=\text { slihtly poor }, l_{4}=\right.\text { fair } \\
& \left.l_{5}=\text { slihtly good }, l_{6}=\text { poor }, l_{7}=\text { very poor }, l_{8}=\text { extremely } \text { good }\right\}
\end{aligned}
$$

By the three decision makers $e_{k}(k=1,2,3)$ (whose weighting vector $\left.v=(0.35,0.25,0.4)^{T}\right)$ under the above four attributes $C_{j}(j=1,2,3,4)$ and decision matrix $M_{k}=\left(l_{i j}^{k}\right)_{5 \times 4},(k=1,2,3)$ are listed as follows:

$$
M_{1}=\left[\begin{array}{llll}
l_{4} & l_{5} & l_{3} & l_{2} \\
l_{8} & l_{7} & l_{7} & l_{4} \\
l_{4} & l_{5} & l_{5} & l_{6} \\
l_{8} & l_{6} & l_{3} & l_{2} \\
l_{6} & l_{4} & l_{7} & l_{5}
\end{array}\right], M_{2}=\left[\begin{array}{llll}
l_{4} & l_{5} & l_{2} & l_{2} \\
l_{6} & l_{6} & l_{6} & l_{3} \\
l_{5} & l_{4} & l_{4} & l_{5} \\
l_{7} & l_{6} & l_{2} & l_{4} \\
l_{5} & l_{3} & l_{6} & l_{4}
\end{array}\right], M_{3}=\left[\begin{array}{llll}
l_{4} & l_{6} & l_{2} & l_{2} \\
l_{6} & l_{4} & l_{5} & l_{5} \\
l_{5} & l_{5} & l_{7} & l_{6} \\
l_{7} & l_{5} & l_{6} & l_{3} \\
l_{5} & l_{7} & l_{6} & l_{6}
\end{array}\right]
$$


Step1. Take the linguistic scale function $\tilde{u}\left(l_{x}\right)=0.8^{x}$ and obtain the function $f^{-1}=\log _{0.8} x$.

Step 2. Calculate the group's decision matrix $G$ by Eq.(4.1). Obtain the group's comprehensive linguistic decision matrix $\tilde{G}$ and the value supporting matrix $\tilde{G}_{S}$.

$$
\tilde{G}=\left[\begin{array}{llll}
l_{4} & l_{5} & l_{2} & l_{2} \\
l_{7} & l_{5} & l_{6} & l_{4} \\
l_{5} & l_{5} & l_{5} & l_{6} \\
l_{7} & l_{6} & l_{4} & l_{3} \\
l_{5} & l_{5} & l_{6} & l_{5}
\end{array}\right], \tilde{G}_{S}=\left[\begin{array}{llll}
4.0000 & 5.3737 & 2.3252 & 2.0000 \\
6.6035 & 5.3542 & 5.8675 & 4.3810 \\
4.6241 & 4.7283 & 5.3810 & 5.7283 \\
7.3252 & 5.5729 & 3.6380 & 2.8350 \\
5.3252 & 4.6380 & 6.3252 & 5.0791
\end{array}\right]
$$

Step 3. Calculate the value supporting of $N_{A_{i}}$ each alternative by Eq.(4.4):

$$
N_{A_{1}}=3.8085, N_{A_{2}}=6.1376, N_{A_{3}}=5.5952, N_{A_{4}}=5.5351, N_{A_{5}}=5.8121 \text {. }
$$

Moreover, the overall assessment $L_{A_{i}}$ of each alternative can be calculated by Figure. 1

$$
\begin{gathered}
L_{A_{1}}=\left(l_{4}, 3.8085\right), L_{A_{2}}=\left(l_{6}, 6.1376\right), L_{A_{3}}=\left(l_{6}, 5.5952\right), \\
L_{A_{4}}=\left(l_{6}, 5.5351\right), L_{A_{5}}=\left(l_{6}, 5.8121\right) .
\end{gathered}
$$

Step 4. Rank the value supporting $N_{A_{i}}(i=1,2, \ldots, 5)$ of each alternative:

$$
N_{A_{2}}>N_{A_{5}}>N_{A_{3}}>N_{A_{4}}>N_{A_{1}} \text {. }
$$

By Eq. (2.6) we can rank all the alternatives $A_{i}(i=1,2, \ldots, 5)$ :

The best alternative is $A_{2}$.

$$
A_{2} \succ A_{5} \succ A_{3} \succ A_{4} \succ A_{1}
$$

\subsection{Comparative and Discussion}

To test the validity of the proposed linguistic MAGDM method, the comparisons are made between the proposed approach and the existing methods ( $\mathrm{Xu}$ ' method [17] and Perk' method [21]). The analysis is based on the above illustrative example.

(1)When taking the linguistic scale function $\tilde{u}\left(l_{x}\right)=x / 8(x \in[0,8])$, the linguistic label $L$ is equidistant. Using the proposed approach, the rank ordering among the alternatives is

$$
A_{2} \succ A_{5} \succ A_{3} \succ A_{4} \succ A_{1} \text {. . }
$$

Using the Park' method [21], the overall assessments of all alternatives can be obtained:

$$
A_{1}=\left(l_{3},-0.193\right), A_{2}=\left(l_{5}, 0.255\right), A_{4}=\left(l_{5},-0.196\right), A_{4}=\left(l_{5},-0.407\right), A_{5}=\left(l_{2}, 0.008\right)
$$

And the rank ordering among the alternatives is $A_{2} \succ A_{5} \succ A_{3} \succ A_{4} \succ A_{1}$.

Obviously, the proposed approach and the Park' method have the same ranking results; therefore, the proposed method is rational and valid.

From above example, when taking the linguistic scale function $\tilde{u}\left(l_{x}\right)=0.8^{x}$, the absolute deviation between the adjacent linguistic subscripts increases, so the linguistic label $L$ is nonequidistant. In such a case, as Herrera and Martinez themselves said that the 2-tuple linguistic representation model was unable to deal with the non-equidistant labels [9]; however, the approach which we proposed is able to overcome this limitation.

(2)Using Xu' method [20], the overall assessments of all alternatives can be obtained: 


$$
A_{1}=l_{3.82,} A_{2}=l_{6.2875}, A_{3}=l_{5.62}, A_{4}=l_{5.6325}, A_{5}=l_{5.925} .
$$

And the rank among the alternatives is $\succ A_{2} \succ A_{5} \succ A_{3} \succ A_{4} \quad A_{1}$.

Although the selection order is the same, the results of $\mathrm{Xu}^{\prime}$ method [17] may not be out of the initial term sets. For example, $l_{3.82} \notin L$ is the virtual linguistic term which only appears in comparison and in operation, then $l_{3.82}$ does not have any syntax or semantics assigned [1]. In the proposed approach, based on the conversion relationship, the linguistic multiset is used to express the overall assessment of each alternative (eg. $L_{A_{1}}=\left(l_{4}, 3.8085\right), l_{4} \in L$ ), which contains the linguistic evaluation of the alternative and the value supporting of the alternative, that can make the aggregation results match with the initial linguistic terms; therefore, the approach which we proposed is more exactly in dealing with linguistic MAGDM problems than the Park' method and the $\mathrm{Xu}$ ' method.

\section{Conclusion}

We provided a linguistic weighted aggregation $L W A_{\varphi}$ operator and studied its properties. The main advantages of the proposed operator are that it can be used to aggregate linguistic variables with equidistant and non-equidistant labels, and it can make the aggregation results to match with the initial linguistic terms. Based on the $L W A_{\varphi}$ operator and the conversion relationship, we obtained a new linguistic MAGDM approach. Finally, an illustrative example was given and some comparisons were made to show the applicability and effectiveness of the proposed approach.

\section{References}

[1] J.Q. Wang, J. Wang, Q.H. Chen, H.Y. Zhang, X.H. Chen. An outranking approach for multicriteria decision making with hesitant fuzzy linguistic term sets[J]. Inf. Sci. 280(1): 338-351(2014)

[2] J.B. Lan, Q. Sun, Q. Chen, Z.X. Wang. Group decision making based on induced uncertain linguistic OWA operators[J]. Decis. Support Syst. 55(1): 296-303(2013)

[3] R. Degani, G. Bortolan. The problem of linguistic approximation in clinical decision making[J]. Int. J. Approx. Reasoning. 2(2): 143-162(1988)

[4] J.H. Hu, Y. Zhang, X.H. Chen, Y.M. Liu. Multi-criteria decision-making method based on possibility degree of interval type-2 fuzzy number [J]. Knowl-Based Syst. 43(2): 21-29(2013)

[5] J.Q. Wang, J.T. Wu, J. Wang, H.Y. Zhang, X.H. Chen. Interval-valued hesitant fuzzy linguistic sets and their applications in multi-criteria decision-making problems[J]. Inf. Sci. 288(20): 5572(2014)

[6] M. Delgado, J.L. Verdehay, M.A. Vila. On aggregation operations of linguistic labels[J]. Int. J. Intell. Syst. 8(3): 351-370(1993)

[7] C.Q. Tan, D.S. Wu, B.J. Ma. Group decision making with linguistic preference relations with application to supplier selection[J]. Expert Syst. 38(12): 14382-14389(2011)

[8] F. Herrera, L. Martínez. The 2-tuple linguistic computational model: advantages of its linguistic description, accuracy and consistency[J]. Int. J. Fuzz. Knowl-Based Syst. 9(1): 33-48(2001)

[9] F. Herrera, L. Martínez. A 2-tuple fuzzy linguistic representation model for computing with words [J]. IEEE Trans. Fuzzy Syst. 8(6): 746-752(2000) 
[10]F. Herrera, E.H. Viedma, L. Martínez. A Fuzzy Linguistic Methodology to Deal With Unbalanced Linguistic Term Sets[J]. IEEE Trans. Fuzzy Syst. 16 (2): 354-370(2008)

[11] J. Wang, J. Hao. An approach to computing with words based on canonical characteristic values of linguistic labels [J]. IEEE Trans. Fuzzy Syst. 15(4): 593-604(2007)

[12] M. Abchir, I. Truck. Towards an extension of the 2-tuple linguistic model to deal with unbalanced linguistic term sets[J]. Kybernetika. 49 (1): 164-180(2013)

[13]Z.S. Xu. Linguistic Decision Making: Theory and Methods[M]. Science Press, Beijing. 3738(2012)

[14]Z.S. Xu. A method based on linguistic aggregation operators for group decision making with linguistic preference relation[J]. Inf. Sci. 166(1-4): 19-30(2004)

[15]Z.S. Xu. Uncertain linguistic aggregation operators based on approach to multiple attribute group decision making under uncertain linguistic environment [J]. Inf. Sci. 168(1-4): 171-184(2004)

[16]Z.S. Xu. Group decision making based on multiple types of linguistic preference relations[J]. Inf. Sci. 178(2): 452-467(2008)

[17]Z.S. Xu. On generalized induced linguistic aggregation operator[J]. Int. J. Gen. Syst. 35(1): 1728(2006)

[18] L.G. Zhou, H.Y. Chen. A generalization of the power aggregation operators for linguistic environment and its application in group decision making[J]. Know-Based Syst. 26(1): 216224(2012)

[19] J.M. Merigó, A.M. Gil-Lafuente, L.G. Zhou, H.Y. Chen. Induced and linguistic generalized aggregation operators and their application in linguistic group decision making[J]. Group Decis. Negot. 21(4): 531-549(2012)

[20]Z.S. Xu. A simulation-based evaluation of several scales in the analytic hierarchy process[J], Systems Engineering-Theory \& Practice. 20(7): 58-62(2000)

[21] G.W. Wei. Some generalize aggregation operators with linguistic information and their application to multiple attribute group decision making[J]. Computer \& Industrial Engineering. 61(1): 32-38(2011) 